\title{
Energetics, Ionization, and Expansion Dynamics of Atomic Clusters Irradiated with Short Intense Vacuum-Ultraviolet Pulses
}

\author{
B. Ziaja, ${ }^{*}$ H. Wabnitz, F. Wang, and E. Weckert \\ Hamburger Synchrotronstrahlungslabor, Deutsches Elektronen-Synchrotron, Notkestrasse 85, D-22607 Hamburg, Germany \\ T. Möller \\ Institut für Optik und Atomare Physik, Technische Universität Berlin, 10623 Berlin, Hardenbergstrasse 36, Germany
}

(Received 21 October 2008; published 19 May 2009)

\begin{abstract}
Kinetic equations are used to model the dynamics of Xe clusters irradiated with short, intense vacuumultraviolet pulses. Various cluster size and pulse fluences are considered. It is found that the highly charged ions observed in the experiments are mainly due to Coulomb explosion of the outer cluster shell. Ions within the cluster core predominantly recombine with plasma electrons, forming a large fraction of neutral atoms. To our knowledge, our model is the first and only one that gives an accurate description of all of the experimental data collected from atomic clusters at $100 \mathrm{~nm}$ photon wavelength.
\end{abstract}

Atomic clusters are excellent objects to test the dynamics within samples irradiated by radiation from short wavelength free-electron-lasers (FELs) [1-3]. Their physical properties put them on the border between the solid state and the gas phase. Cluster studies are important for planned experiments with FELs in solid state physics, materials science, and for studies of the extreme states of matter [4]. Accurate predictions of ionization, thermalization, and expansion time scales within irradiated samples, which may be obtained with cluster experiments [5-9], are also needed for exploring the limits of experiments for single particle diffraction imaging [10-15].

During the first cluster experiments performed at the FEL facility FLASH at DESY with VUV photons of energy, $E=12.7 \mathrm{eV}$, and power densities up to a few $10^{13} \mathrm{~W} / \mathrm{cm}^{2}$ [5] energetic, highly charged Xe ions were detected. The estimated energy absorption was almost an order of magnitude higher than that predicted with classical absorption models [6-8], and the ion charge states created were much higher than those observed during the irradiation of isolated atoms in similar conditions. This indicated that at these wavelengths some processes specific to many-body systems were responsible for an enhanced energy absorption.

The physics underlying the dynamics within the irradiated clusters is complex. Several theoretical models have been proposed in order to describe the evolution of clusters exposed to intense VUV pulses [16-22]. These include (i) heating of quasifree electrons due to enhanced inverse bremsstrahlung (IB) [16,17], (ii) enhanced photoionization within the sample due to a lowering of the interatomic potential barriers $[18,19]$, and (iii) heating due to manybody recombination processes [21]. Each of these various approaches leads in a different way to a significantly enhanced energy absorption in agreement with the experimental data [5].
In order to evaluate the contribution of various processes to the ionization dynamics, including the enhancement factors proposed in Refs. [16-19,21], we have constructed a unified model $[23,24]$ based on kinetic equations. It included the following predominant interactions: photoand collisional ionization, elastic electron-ion and electron-atom scattering, IB heating of quasifree electrons, shifts of energy levels within atomic potentials due to the plasma environment, and shielded electron-electron interactions. IB rates were calculated as in Refs. [16,17], using the effective atomic potentials. This led to the strong enhancement of these rates, comparing to the approaches that used the pointlike Coulomb potentials, e.g., $[25,26]$. The calculated IB rates were included explicitly in the kinetic equations in the form of the additional source terms.

These interactions were discussed in detail in Ref. [23]. We do not include atom-ion, ion-ion and atom-atom collisions to our model, as their rates are small comparing to the electron-ion(atom) collision rates.

Using a nonequilibrium Boltzmann solver we followed the evolution of xenon clusters of various size irradiated with rectangular shaped VUV pulses of intensities within the limits: $10^{12}-10^{14} \mathrm{~W} / \mathrm{cm}^{2}$ and pulse duration between 10 and 50 fs. We found that each physical mechanism included in the model contributed to the ionization dynamics, however, with differing weights. The total ionization rate within the sample was most strongly affected by the IB heating rate applied. Within the theoretical framework defined above [23] we estimated that (i) many-body recombination (four-body and higher) [21] could contribute only for clusters irradiated at low pulse fluences, and (ii) the plasma environment effects estimated with electron screened atomic potentials were small. This was in contrast to the estimates of Ref. [18] performed with unscreened atomic potentials. Our studies $[23,24]$ extended the treat- 
ment of Ref. [17], where neither the spatial inhomogeneity of the clusters was treated nor the gross movement of electrons. It arises that both effects significantly contribute to the cluster dynamics. The structure of charge created within the cluster was found experimentally to be strongly inhomogeneous [27], and this inhomogeneity was induced by the dynamics of the electrons.

The main aim of this Letter is to obtain a complete description of the energetics, ionization and expansion dynamics of irradiated clusters. Our present analysis differs from the analysis in $[23,24]$ by including the effect of three-body recombination and following the expansion of the cluster until ions begin to leave the simulation box (up to $\sim 2 \mathrm{ps}$ ). In other words, we now follow the dynamics starting from the geometry of dense, neutral clusters up to a dilute gas of isolated ions, electrons, and neutral atoms.

The three-body recombination is the inverse of the collisional ionization, and this relation is used here in deriving the relevant rates. Including the three-body recombination into our code, we take into account the fact that the first phase of the cluster evolution is nonequilibrium. Therefore, we follow the approach of Ref. [28] and estimate the three-body recombination contribution, assuming an arbitrary electron distribution. This approach is appropriate both for the nonequilibrium and the equilibrium evolution phase. The recombination rates are then estimated, using the microscopic reciprocity of differential cross sections for collisional ionization and recombination [29].

The results from the model are averaged over the spatial pulse profile. Furthermore, they are also cross-checked with independent molecular dynamics (MD) simulations. This leads to the following observations:

(i) In the previous work $[23,24]$ the hypothesis was proposed that the dominant contribution to the observed ion spectra may come from the outer shell of the irradiated cluster. In order to justify this hypothesis, the inclusion of the recombination within the cluster, following its expansion and averaging the results obtained over the spatial pulse profile was necessary. Only then could the predictions obtained with the model be compared to those recorded by the time-of-flight detector during the experiment.

(ii) The impact of three-body recombination on the ionization dynamics is very important. In particular, it has changed the estimated ion charge distribution within the ionized cluster. In contrast with the results of Refs. [23,24] the highest ion charges are now created in the outer shell of the cluster and not within the cluster core. A large fraction of neutral atoms could also be found within the cluster core. The proportion is dependent on the cluster size and pulse fluence.

(iii) Including the cluster expansion has also allowed us to observe the differing expansion dynamics for both small and large clusters.

(iv) We have tested our model with an independent, dedicated molecular dynamics simulation for smaller clusters. The results obtained from both methods are in good agreement. This confirms that our analysis was not biased by our choice of simulation method.

The dynamics within the irradiated clusters depends on the pulse fluence and the cluster size. We have performed simulations of irradiated clusters with parameter values corresponding to those at the first FLASH cluster experiment at $100 \mathrm{~nm}$ wavelength $[5,8]$. Below we compare the predictions to the experimental data.

The simulations were performed for clusters exposed to single rectangular shaped VUV pulses of a fixed fluence. The temporal VUV FEL pulse shape varies from shot to shot. We accounted for that approximately by performing a number of simulations with different pulse lengths at each value of the fluence. The intensity was set to the fluence divided by the pulse length. For the quoted ranges of pulse duration and a fluence range of 0.05 to $1.5 \mathrm{~J} / \mathrm{cm}^{2}$, the full intensity range was $10^{12}-1.5 \times 10^{14} \mathrm{~W} / \mathrm{cm}^{2}$. The predictions obtained were then averaged over the number of simulated shots. This scheme followed the experimental data analysis $[5,7]$. We estimate that the real value of pulse fluence may be up to 1.5 times smaller and up to 2 times larger than that one estimated experimentally. This asymmetry is due to systematic errors in the fluence estimation.

We obtain predictions for two limiting cases: (i) a cluster placed in the center of the focused beam and (ii) the position of the cluster integrated over the approximately estimated spatial profile of the pulse. As we will see later, at higher pulse fluences averaging over the spatial pulse profile has a higher impact on the model predictions compared to the case of a cluster placed in the center of the focused beam. This is due to the strong nonlinear dependence of the ion charge created on pulse fluence, at higher pulse fluences. At lower fluences this dependence is linear.

First, we will investigate ionization dynamics at different cluster sizes. In Figs. 1(a)-1(d) we plot the charge state distributions at the end of the expansion phase obtained for four different cluster sizes irradiated with a fixed pulse fluence of $0.4 \mathrm{~J} / \mathrm{cm}^{2}$. The trend can be understood in the following way. IB is the dominant mechanism of energy absorption within our model. This process heats up the quasifree electrons within the cluster. The hot electrons collide with ions and atoms, producing higher charges and releasing new electrons. These processes compete with the three-body recombination processes that reduce the ion charge and decrease the electron density. After the pulse is over, and the system reaches a local thermodynamic equilibrium (LTE) state, the number of ionization and recombination events saturates, changing slowly with the decreasing temperature within the cluster. We find that during the exposure the cluster forms a shell structure [27] consisting of a positively charged outer shell and a core of net charge equal to zero. Such a shell structure has been previously found by other groups in the context of $\mathrm{x}$-ray irradiation of clusters and biomolecules [18,30,31]. The width of these shells depends on the cluster size. The charged outer shell is large within small clusters $\left(N_{\text {atoms }}=\right.$ 20,70), and its Coulomb explosion drives the expansion of 


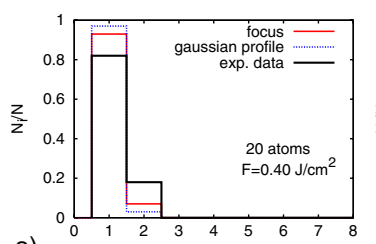

a)

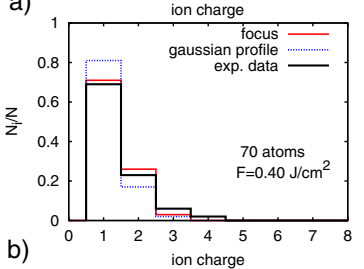

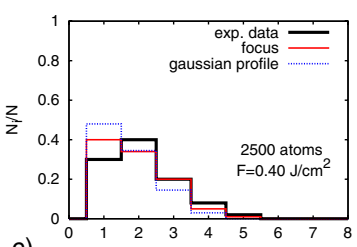

C) $\begin{array}{llllll}0 & 1 & 2 & 3 & 4 & 5 \\ \text { ion charge }\end{array}$

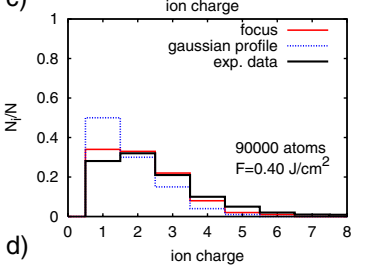

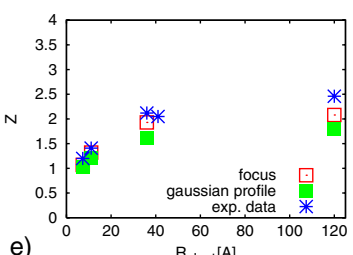

e)

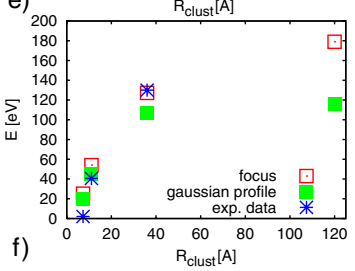

FIG. 1 (color online). Ion fractions, $N_{i} / N$, within the irradiated clusters estimated for four various cluster sizes. $N_{i}$ denotes here the number of $\mathrm{Xe}^{+i}$ ions, and $N$ is the total number of ions. We show also: (e) average charge, $Z$, created within the irradiated cluster, and (f) average energy absorbed per ion, $E$, as a function of the cluster radius, $R$. Experimental data, model predictions for clusters placed in the center of the focussed beam and predictions integrated over the Gaussian spatial pulse profile are shown.

these clusters. Within large clusters $\left(N_{\text {atoms }}=2500\right.$, 90000 ) the neutral core is large, and after the Coulomb explosion of the outer shell it expands hydrodynamically. Highly charged ions within the core recombine efficiently with electrons. As a result, we observe a large fraction of neutral atoms created within the core, the proportion depending on the cluster size. This fraction is from $15 \%$ to $40 \%$ of the total number of particles. Within small clusters a large fraction of the electrons released during the ionization processes can leave the clusters early in the exposure. The remaining electrons are not heated efficiently via IB processes due to their low density within the cluster. Consequently, only low charge states are observed [Figs. 1(a) and 1(b)]. In contrast, within large clusters of 2500 and 90000 xenon atoms only a small fraction of the released electrons are able to escape from the cluster. The width of the positively charged outer shell is small with respect to the radius of the neutral core. Electrons confined within the core are then heated efficiently. This leads to further collisional ionizations. In Fig. 1(c) and 1(d) we plot the respective ion fractions. Our predictions are in agreement with the experimental measurements. The trend of the size dependence is correct. At a fixed pulse energy the maximal and average ion charge created increases with the cluster size until it saturates at larger cluster sizes. This is in agreement with the experimental data [Fig. 1(e)]. Saturation of the ion charge created within large clusters (irradiated with a pulse of a fixed fluence) is due to the fact that for large enough clusters the energy absorbed from the pulse will not be sufficient for the creation of higher charge states. We also show our estimates for the average kinetic energy per ion, $E$, [Fig. 1(f)]. It increases with the cluster size and saturates for larger clusters.

With the extended Boltzmann solver we were able to follow the different expansion dynamics for large and small clusters (not shown due to limitations of space). In the case of small clusters the expansion was driven by the Coulomb explosion, and ions then quickly filled the whole volume of the simulation box. In the case of a dominating hydrodynamic expansion of the sample, the evolution proceeded shell-after-shell as predicted by earlier simulations [13].

The dependence of the ionization dynamics on pulse fluence has been investigated for $\mathrm{Xe}_{2500}$ clusters. Below
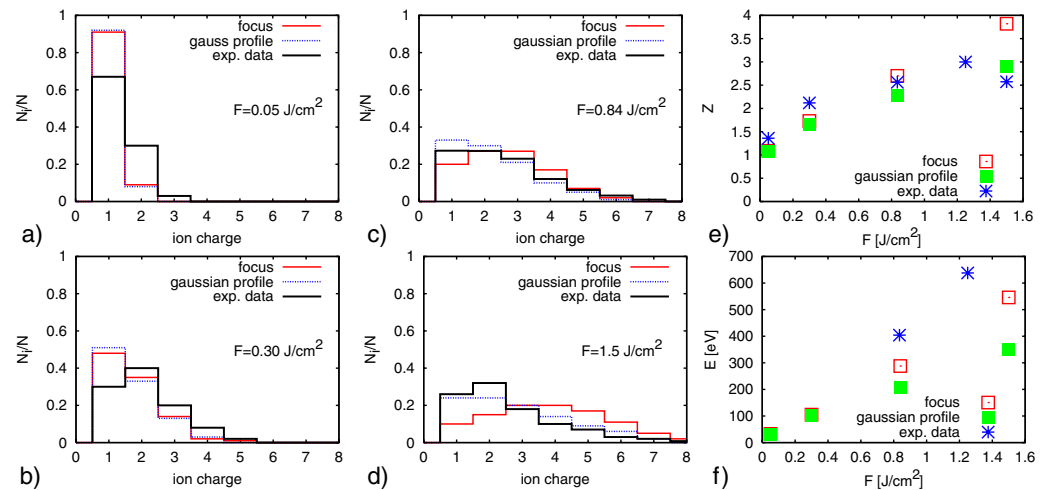

FIG. 2 (color online). Ion fractions, $N_{i} / N$, within the irradiated xenon clusters $\left(N_{\text {atoms }}=2500\right)$. These clusters were irradiated with rectangular shaped pulses of four various fluences. We show also: (e) average charge, $Z$, created within the irradiated xenon cluster, and (f) average energy absorbed per ion, $E$, as a function of the pulse fluence, $F$. Other notations are as in Fig. 1. 
we show the plots of the ion fractions [Figs. 2(a)-2(d)]. Higher pulse fluences lead to the creation of higher charge states within the clusters in accordance with the mechanism described above. At the lower pulse fluences, $F=$ $0.05-0.3 \mathrm{~J} / \mathrm{cm}^{2}$, the small discrepancy between experiment and our predictions may be due to the fact that an additional energy absorption due the many-body recombination effects within the cold electron plasma can occur [23]. This could then lead to the creation of higher charge states that we do not observe within our model, as manybody recombination [21] was not included into it. At higher fluences, $F=0.84-1.5 \mathrm{~J} / \mathrm{cm}^{2}$, the ion fractions are in good agreement with experimental data.

The average charge is plotted as a function of pulse fluence in Fig. 2(e). With increasing pulse fluence the average charge created increases. The charges calculated are very close to the corresponding experimental values. Also, as expected, the content of neutral atoms found within the cluster decreases at the increasing pulse fluence: from $70 \%$ to $5 \%$ for fluences: $F=0.05-1.5 \mathrm{~J} / \mathrm{cm}^{2}$ (not shown). We also show the average kinetic energy per ion as a function of the pulse fluence [Fig. 2(f)]. The sparse experimental data do not allow the identification of the trend of the fluence dependence, i.e., whether it is linear or nonlinear. Our predictions slightly underestimate the experimental predictions for ion energy at high fluences but stay within the error limit given by the experimental uncertainty of fluence estimation.

In summary, we used a microscopic Boltzmann model to investigate dynamics within atomic clusters irradiated by single VUV pulses. The predictions obtained at various cluster sizes and pulse fluences were found to be in good overall agreement with experimental data considering the uncertainty of the pulse fluence estimation. The results obtained have been successfully cross-checked with MD simulations showing that they are not biased by the choice of our particular simulation method. Because of limitations of space we do not discuss the MD results here. The present analysis shows that recombination contributes significantly to the ionization dynamics. As expected, the recombination has the highest impact within the cluster core where the electron density is highest. This indicates that the ions detected during the experiment originate mainly from the outer region of the cluster [27]. We found a large fraction of neutrals exist within the core resulting from the recombination, the proportion depending on the cluster size. This prediction is especially important, as neutral fragments can be detected in an experiment only with a great difficulty. If we ignore them, the total energy absorption due to the laser-cluster interaction may be extensively overestimated.

In conclusion, we find that formation of high charge states and the strong absorption of VUV radiation is quantitatively understood within the framework of our model.

B. Ziaja is grateful to C. Bostedt, H. Chapman, C. Deiss, T. Laarmann, A. Mancuso, W. Rozmus, R. Santra, and A. Szöke for illuminating comments. Authors thank the colleagues from Dresden, especially J.M. Rost and U.
Saalmann, as well as J. Jortner (Tel Aviv) for fruitful discussions. This research was supported by the German Bundesministerium für Bildung und Forschung with Grants No. 05 KS4 KTC/1, 05 KS7 KT1 and by the Helmholtz Gemeinschaft, Impulsfond VH-VI-302.

*Corresponding author. beata.ziaja-motyka@desy.de

Also at Institute of Nuclear Physics, Radzikowskiego 152, 31-342 Krakow, Poland.

[1] DESY. Technical Design Report of the European XFEL, ISBN 3-935702-17-5 5, 7-9 2006.

[2] LCLS. LCLS: The First Experiments., SSRL, SLAC, Stanford, USA, 2000.

[3] T. Shintake and (SCSS Team), Proceedings of FEL 2006 BESSY, Berlin, Germany (BESSY, Berlin, 2006), p. 33.

[4] DESY. XFEL-info. DESY, http://xfelinfo.desy.de 5, p. 18 2007.

[5] H. Wabnitz et al., Nature (London) 420, 482 (2002).

[6] J. Schulz et al., Nucl. Instrum. Methods Phys. Res., Sect. B 507, 572 (2003).

[7] H. Wabnitz, Ph.D. thesis, DESY-THESIS-2003-026, 2003.

[8] T. Laarmann et al., Phys. Rev. Lett. 92, 143401 (2004).

[9] C. Bostedt et al., Phys. Rev. Lett. 100, 133401 (2008).

[10] R. Neutze et al., Nature (London) 406, 752 (2000).

[11] J. Miao, K. Hodgson, and D. Sayre, Proc. Natl. Acad. Sci. U.S.A. 98, 6641 (2001).

[12] Z. Jurek, G. Oszlányi, and G. Faigel, Europhys. Lett. 65, 491 (2004).

[13] S. P. Hau-Riege et al., Phys. Rev. E 71, 061919 (2005).

[14] S. P. Hau-Riege et al., Phys. Rev. Lett. 98, 198302 (2007).

[15] H. Chapman et al., Nature Phys. 2, 839 (2006).

[16] R. Santra and C. H. Greene, Phys. Rev. Lett. 91, 233401 (2003).

[17] Z. B. Walters, R. Santra, and C.H. Greene, Phys. Rev. A 74, 043204 (2006).

[18] C. Siedschlag and J. M. Rost, Phys. Rev. Lett. 93, 043402 (2004).

[19] I. Georgescu, U. Saalmann, and J.-M. Rost, Phys. Rev. A 76, 043203 (2007).

[20] D. Bauer, J. Phys. B 37, 3085 (2004).

[21] C. Jungreuthmayer et al., J. Phys. B 38, 3029 (2005).

[22] M. Rusek and A. Orlowski, Phys. Rev. A 71, 043202 (2005).

[23] B. Ziaja, H. Wabnitz, E. Weckert, and T. Möller, New J. Phys. 10, 043003 (2008).

[24] B. Ziaja, H. Wabnitz, E. Weckert, and T. Möller, Europhys. Lett. 82, 24002 (2008).

[25] V.P. Krainov and M. B. Smirnov, Phys. Rep. 370, 237 (2002).

[26] V. P. Krainov. J. Phys. B 33, 1585 (2000).

[27] M. Hoener et al., J. Phys. B 41, 181001 (2008).

[28] H. K. Chung et al., High Energy Density Phys. 1, 3 (2005).

[29] J. Oxenius, Kinetic Theory of Particles and Photons (Springer-Verlag, Berlin, 1986), p. 21.

[30] Z. Jurek, G. Faigel, and M. Tegze, Eur. Phys. J. D 29, 217 (2004).

[31] S. P. Hau-Riege, R. A. London, and A. Szöke, Phys. Rev. E 69, 051906 (2004). 